\title{
Symmetry-protected quantization and bulk-edge correspondence of massless Dirac fermions: Application to the fermionic Shastry-Sutherland model
}

\author{
Toshikaze Kariyado* and Yasuhiro Hatsugai \\ Division of Physics, Faculty of Pure and Applied Sciences, University of Tsukuba, Tsukuba, Ibaraki 305-8571, Japan
}

(Received 30 July 2013; published 20 December 2013)

\begin{abstract}
Berry phases defined by the one-dimensional momentum as a parameter show $Z_{2}$ quantization due to the inversion symmetry combined with the time reversal, or existence of the reflection plane, which also protects the massless Dirac cones with continuous parameters. This is the symmetry protected $Z_{2}$ quantization. These ideas are applied in the fermionic Shastry-Sutherland model, which has a rich phase diagram, including phases with massless Dirac fermions, a quadratic band crossing point, and a pseudospin-1 Weyl fermion. We also demonstrate that the $Z_{2}$ Berry phases determine the existence of edge states as the bulk-edge correspondence of the massless Dirac fermion systems.
\end{abstract}

DOI: 10.1103/PhysRevB.88.245126

PACS number(s): 73.20.-r, 03.65.Vf, 71.10.Pm

\section{INTRODUCTION}

Massless Dirac fermion systems, which are zero gap semiconductors characterized by a linear dispersion, exhibit many intriguing phenomena. ${ }^{1-5}$ A typical realization of massless Dirac fermion system is celebrated graphene, ${ }^{1}$ which has been attracted much attention since its discovery. Not only in conventional solid state materials, but also in optical lattice systems, the fabrication of massless Dirac fermions becomes a hot topic. ${ }^{6,7}$ Among the many unusual properties of the massless Dirac fermions, appearance of characteristic edge states $^{8-10}$ is important in the view of the bulk-edge correspondence, which implies that topologically nontrivial bulk states and appearance of the edge states are closely related and reflect each others. The concept "bulk-edge correspondence" is established for a topologically nontrivial gapped state. ${ }^{11}$ There, a bulk topological number and number of edge states are connected. Although the massless Dirac fermion system is gapless at the Fermi energy and a topological number cannot be well defined, the bulk-edge correspondence is still at work..$^{9,12,13}$

Instead of the bulk topological number such as the Chern number, the Berry phase $\theta\left(k_{\|}\right)$plays a central role in the massless Dirac fermion systems. ${ }^{9,12,13}$ Here, $\theta\left(k_{\|}\right)$is a bulk quantity parameterized by a momentum $k_{\|}$, which is parallel to the "edge." Generically, the Berry phase $\theta\left(k_{\|}\right)$is gauge dependent and takes any real number in modulo $2 \pi$ in contrast to the Chern number that is gauge invariant and intrinsically integer. ${ }^{14}$ However, with the help of a supplemental symmetry, the Berry phase is quantized and becomes topological, that is, adiabatic invariant. ${ }^{12,15-18}$ This is the symmetry protected quantization. At the same time, the symmetry further plays a crucial role for the topological stability of the massless Dirac fermions. Since the gap closing point has co-dimension $3,{ }^{17,19}$ the symmetry discussed above is crucial to have a massless Dirac fermions in two-dimensions in a generic situation. Practically, the chiral symmetry has been often employed to discuss the quantization of the Berry phase, the bulk-edge correspondence, and the stability of the massless Dirac fermions. ${ }^{9,12}$

In this paper, we show that the Berry phase is generally quantized into $Z_{2}$ values by the inversion symmetry combined with the time-reversal symmetry or the reflection symmetry.
Also, intimate relation between the quantized Berry phase $\left(Z_{2}\right.$ Berry phase) and the stability of the massless Dirac fermions in two dimensions is discussed without using the chiral symmetry. Further, we demonstrate that the $Z_{2}$ Berry phase determines the existence of the edge modes, so as to discuss the bulk-edge correspondence in the massless Dirac fermion systems. For clarity, the ideas are explained taking the fermionic Shastry-Sutherland (SS) model as an example. This model has not been studied in detail, while a spin model on the SS lattice, which is known as the orthogonal dimer model, has been extensively studied after the discovery of the exact ground state ${ }^{20-23}$ and its experimental realization. ${ }^{24}$ We find that the fermionic SS model, which naturally breaks the chiral symmetry, has a rich phase diagram containing the phases with massless Dirac fermions, a quadratic band crossing point, ${ }^{25}$ or a pseudospin-1 Weyl fermion ${ }^{26,27}$ (also known as SO(3) Dirac electron ${ }^{28}$ ). In the following, we begin with describing the fermionic SS model (see Sec. II) and then describe $Z_{2}$ Berry phases (see Sec. III).

\section{FERMIONIC SHASTRY-SUTHERLAND MODEL}

\section{A. Hamiltonian}

A possible physical realization of Shastry-Sutherland lattice and its schematic picture are shown in Figs. 1(a) and 1(b). This lattice possesses many symmetries among which the fourfold rotational symmetry, the glide plane symmetry, and the inversion symmetry play particularly important roles in the following arguments. A shaded region represents a unit cell, which contains four lattice sites named site 1-4, implying that the model has four bands. The Hamiltonian is

$$
H=\sum_{a b} \sum_{\boldsymbol{r} \boldsymbol{r}^{\prime}} t_{a b}\left(\boldsymbol{r}-\boldsymbol{r}^{\prime}\right) c_{\boldsymbol{r} a}^{\dagger} c_{\boldsymbol{r}^{\prime} b}=\sum_{a b \boldsymbol{k}}\left(\hat{H}_{\boldsymbol{k}}\right)_{a b} c_{\boldsymbol{k} a}^{\dagger} c_{\boldsymbol{k} b},
$$

with $c_{\boldsymbol{k} a}=\frac{1}{\sqrt{N}} \sum_{\boldsymbol{r}} \mathrm{e}^{\mathrm{i} \boldsymbol{k} \cdot \boldsymbol{r}} c_{\boldsymbol{r} a}$. Here, indices $a$ and $b$ run from 1 thorough 4, representing four sublattices, while $\boldsymbol{r}$ and $\boldsymbol{r}^{\prime}$ represents lattice vectors on square lattice. We employ four parameters $t_{+}, t_{-}, t_{x}$, and $t_{y}$ that correspond to transfer integrals between the sites connected by bonds indicated as,+- , $x$, and $y$ in Fig. 1(b), respectively. For convenience, we use parameters $t_{0}, t_{1}, \Delta_{0}$, and $\Delta_{1}$ defined as $t_{ \pm}=t_{0} \pm \Delta_{0}$, 

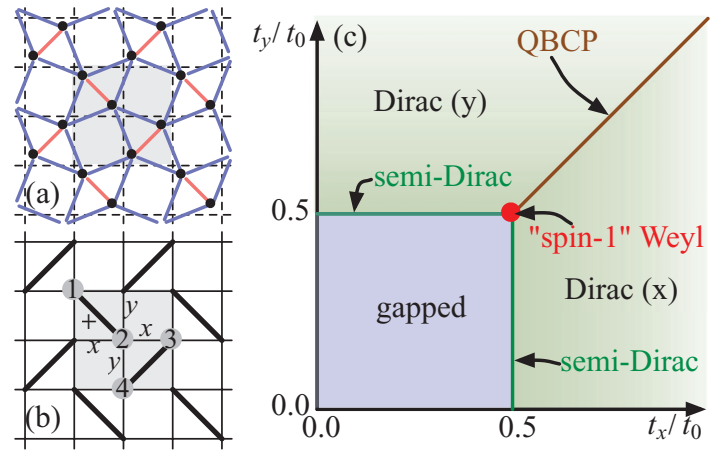

FIG. 1. (Color online) (a) The most "physical" ShastrySutherland lattice. (b) Schematic picture of our model. Bonds named as,,$+- x$, and $y$ are associated with the transfer integrals $t_{+}$, $t_{-}, t_{x}$, and $t_{y}$, respectively. Shaded region denotes the unit cell. We give numbers one through four to four sites in a unit cell in order to distinguish them. (c) Phase diagram for the case of $t_{+}=t_{-}=t_{0}$.

$t_{x}=t_{1}+\Delta_{1}$, and $t_{y}=t_{1}-\Delta_{1}$. The finite $\Delta_{0}$ breaks the glide plane symmetry keeping the reflection symmetry whose reflection plane lies in the diagonal direction, while the finite $\Delta_{1}$ breaks the fourfold rotational symmetry keeping the glide plane symmetry. The inversion symmetry is always maintained with this parametrization. In this study, we neglect spin degrees of freedom, and concentrate on the half filled case. Namely, "gapped state" in the following means that the system has a gap between the second and third lowest bands.

Potential candidates of the material realization for the described model are some variants of the (quasi) twodimensional materials realizing the spin SS model. Naively, $t_{1}<t_{0}$ is expected when the diagonal bonds are shorter than the "rectangular" bonds [see Fig. 1(a)]. The finite $\Delta_{0}\left(\Delta_{1}\right)$ can be induced by applying the uniaxial pressure in the diagonal (rectangular) direction. Actually, the pressure in the diagonal direction induces the changes other than finite $\Delta_{0}$, but the essential physics is captured by finite $\Delta_{0}$ only. The optical lattice systems may be another choice for realizing the proposed model.

\section{B. Phase diagram}

The phase diagram obtained for $\Delta_{0}=0\left(t_{+}=t_{-}\right)$is shown in Fig. 1(c). For $t_{x}=t_{y}<0.5 t_{0}$, the system is in a (trivial) gapped phase. On the other hand, for $t_{x}=t_{y}>0.5 t_{0}$, we find a quadratic band crossing point (QBCP) ${ }^{25}$ at which two parabolic bands, one is holelike and the other is electronlike, touch with each other, at the $\Gamma$ point, ${ }^{21,29}$ [see Figs. 2(a) and 2(d)]. The holelike band is not parabolic in a strict sense in this case, since it is dispersionless in the $\Gamma-M$ direction. QBCP is allowed to exist if the system has a fourfold rotational symmetry, ${ }^{30}$ and has interesting properties. For instance, the fourfold symmetry can be broken by electron-electron interaction effects, leading to emergent nematic phases. ${ }^{25}$ For $t_{x}=t_{y}=0.5 t_{0}$, at which the transition between the trivial gapped phase and the phase with QBCP takes place, there exists "pseudospin-1 Weyl fermion" 27 (also known as $\mathrm{SO}(3$ ) Dirac electron ${ }^{28}$ ), which is characterized by a linear dispersion
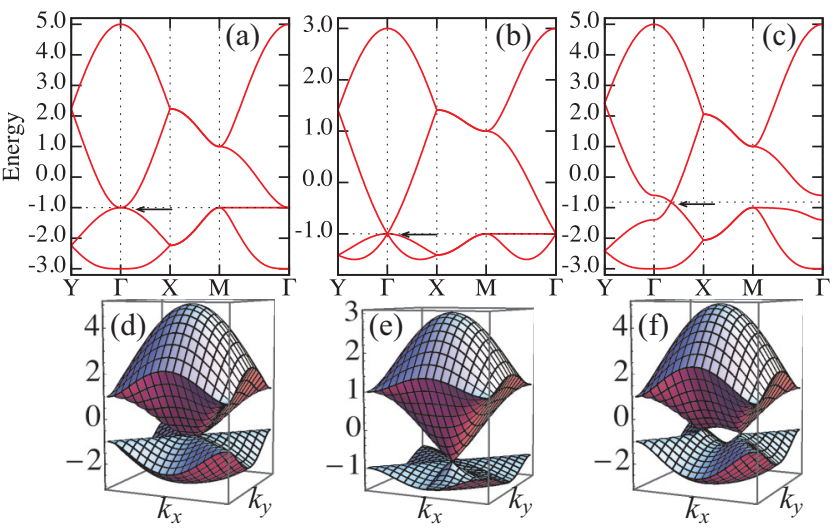

FIG. 2. (Color online) Band structures and dispersion relations. $\left(t_{0}, \Delta_{0}\right)=(1.0,0.0)$ for the all three cases, while $\left(t_{1}, \Delta_{1}\right)$ is $(0.0,0.0)$ [(a) and (d)], $(0.5,0.0)[(\mathrm{b})$ and (e)], and $(1.0,0.1)$ [(c) and (f)]. In (e), a part of the dispersion is eliminated so as to make the inside visible.

and a threefold degeneracy, ${ }^{26}$ at the $\Gamma$ point [see Figs. 2(b) and 2(e)].

A finite $\Delta_{1}\left(t_{x} \neq t_{y}\right)$ imposed in the QBCP phase immediately leads to a phase with Dirac cones at the Fermi energy [see Figs. 2(c) and 2(f)]. Namely, two Dirac cones (and two Dirac points associated with them) are generated as a pair from the QBCP by a finite $\Delta_{1}$. The Dirac points are located on the $k_{x}$ axis for $\Delta_{1}>0$, while they are on the $k_{y}$ axis for $\Delta_{1}<0$. Then, if $\Delta_{1}$ is continuously modified from positive to negative, the Dirac points first move on the $k_{x}$ axis towards the $\Gamma$ point until they merge, and they next depart from the $\Gamma$ point in the direction of the $k_{y}$ axis. Note that the second lowest band is no longer dispersionless on the $\Gamma-M$ direction [see Fig. 2(c)], which is important for letting the Dirac fermions being the only entity appearing at the Fermi energy. If $t_{1}$ is made smaller and smaller with finite $\Delta_{1}$, the system experiences a transition from the phase with Dirac cone to the trivial gapped phase. The transition between two phases is characterized by an appearance of a semi-Dirac fermion, whose dispersion is linear in one direction and parabolic in the other direction. Actually, this type of disappearance of the Dirac cones is rather general and found in many other models for Dirac fermions. ${ }^{31}$ It is also worth noting that for $t_{x}=0$ with $t_{y}=t_{+}=t_{-}$(or $t_{y}=0$ with $t_{x}=t_{+}=t_{-}$), the model can be regarded as a honeycomb lattice model.

When $\Delta_{0} \neq 0$ simultaneously with $\Delta_{1} \neq 0$, the Dirac points go into the general points in the Brillouin zone apart from the high-symmetry lines, i.e., the $k_{x}$ and $k_{y}$ axes. For instance, when $\left(\Delta_{0}, \Delta_{1}\right)$ is changed according to $\left(\Delta_{0}, \Delta_{1}\right)=$ $\left(\delta_{0} \sin \phi, \delta_{1} \cos \phi\right)$ with $t_{0}=t_{1}=1.0$, the Dirac points wind around the $\Gamma$ point as $\phi$ grows from 0 to $2 \pi$. Trajectories of Dirac points for the cases of $\left(\delta_{0}, \delta_{1}\right)=(0.1,0.1)$ and $(0.2,0.1)$ are shown in Fig. 3. The physical state gets back to the original state after $2 \pi$ change in $\phi$, but each Dirac point does not get back to the original position: two Dirac points interchange their position. Remember that when both of $\Delta_{0}$ and $\Delta_{1}$ are finite, the system loses the most of the symmetries, but keeps the inversion (and time-reversal) symmetry, which is essential for stabilizing the Dirac cones. 


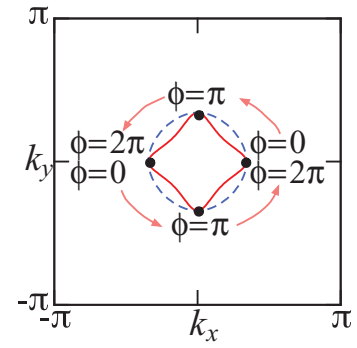

FIG. 3. (Color online) Trajectories of Dirac points for $\left(\delta_{0}, \delta_{1}\right)=$ $(0.1,0.1)$ (solid line) and for $\left(\delta_{0}, \delta_{1}\right)=(0.2,0.1)$ (dashed line).

\section{SYMMETRY PROTECTED $Z_{2}$ BERRY PHASE AND BULK-EDGE CORRESPONDENCE}

\section{A. General theory and application to the fermionic Shastry-Sutherland model}

Now, let us discuss the bulk-edge correspondence of the massless Dirac fermions. For this purpose, we calculate edge spectra and Berry phase for fermionic Shastry-Sutherland model. For simplicity, we discuss the edge parallel to the $x$ axis, but it is possible to extend the following methods to more general cases. ${ }^{13}$ Edge spectra are calculated by making the system with strip (or ribbon) geometries. Here, in order to make a direct connection to the Berry phase arguments, we set a rule to make strips for calculation: edges are given by cutting a periodic system in between the unit cells. With this construction, the edge shapes, or how the system is terminated at the edge, crucially depend on the convention of the unit cell since the position of the cut is fixed in between the unit cells. In this paper, we treat two kinds of unit cell conventions that lead to two kinds of edge terminations, illustrated as Figs. 4(a) and 4(b), respectively. Hereafter, we call the convention in Figs. 4(a) and 4(b) "type 1" and "type 2," respectively. As we limit our attention to the edge parallel to the $x$-axis, Berry phase $^{12,13,15}$ is defined as

$$
\mathrm{i} \theta\left(k_{\|}\right)=\sum_{n \in \text { filled }} \int_{-\pi}^{\pi} \mathrm{d} k_{\perp}\left\langle u_{n k_{\|} k_{\perp}}\left|\partial_{k_{\perp}}\right| u_{n k_{\|} k_{\perp}}\right\rangle,
$$

where $k_{\|}$and $k_{\perp}$ are essentially $k_{x}$ and $k_{y}$, and $\left|u_{n k_{\|} k_{\perp}}\right\rangle$ is a Bloch wave function that is a four-component vector for our
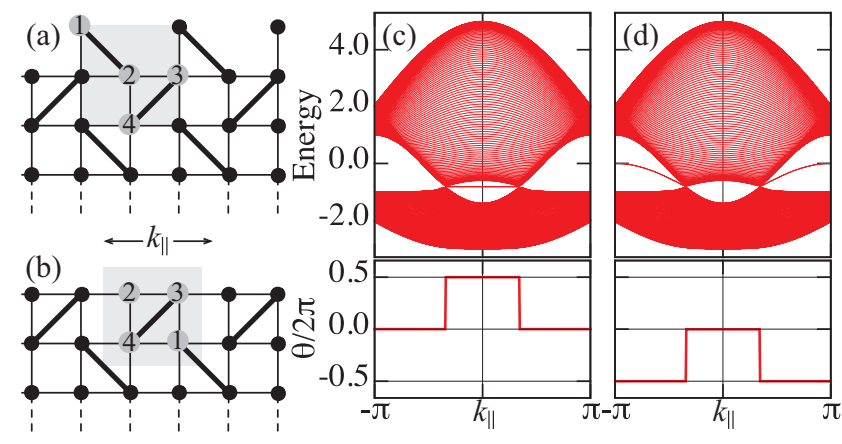

FIG. 4. (Color online) (a) and (b) Unit cell conventions and edge shapes. (c) and (d) Edge spectra and Berry phase $\theta\left(k_{\|}\right)$divided by $2 \pi$ as functions of $k_{\|}$for $\left(t_{0}, t_{1}, \Delta_{0}, \Delta_{1}\right)=(1.0,1.0,0.0,0.1)$. (c) is for type 1 edge (a), while (d) is for type 2 edge (b). four-band tight-binding model. Although we handle a twodimensional model here, the extension to $d$ dimensional cases is straight forward. Namely, we simply regard $k_{\|}$as a $d-1$ dimensional vector rather than a number. Actual evaluation of Eq. (2) is performed using a technique in Refs. 32 and 33.

Calculated edge spectra and $\theta\left(k_{\|}\right) / 2 \pi$ for type 1 and type 2 conventions with $\left(t_{0}, t_{1}, \Delta_{0}, \Delta_{1}\right)=(1.0,1.0,0.0,0.1)$ are plotted as functions of $k_{\|}$in Figs. $4(\mathrm{c})$ and $4(\mathrm{~d})$. From these figures, we can extract three important points: (1) appearance of edge states, (2) quantization of the Berry phase, and (3) an intimate relation between the edge states and the Berry phase. We explain these in turn in the following.

\section{Edge states in the fermionic Shastry-Sutherland model}

Since we chose the parameters so as to have bulk massless Dirac fermions, bulk continuum, which is the filled region in Figs. 4(c) and 4(d) and contributed from the bulk states, becomes gapless at the projected Dirac points. We find the edge states apart from the bulk continuum connecting the projected Dirac points for both of the type 1 and 2 cases. The edge states for the type 1 and 2 cases are different due to the different edge termination. For the type 1 case, the edge state appears near $k_{\|}\left(=k_{x}\right)=0$, while for the type 2 case, it appears near $k_{\|}=\pi$. For both cases, the edge states are dispersive since there is no chiral symmetry that limits the energy of the edge states, but the edge state shows almost flat dispersion for the type 1 case.

\section{Symmetry protected quantization of the Berry phase: general theory}

In general, the quantization of the Berry phase is caused by some symmetry. In the case of Eq. (2), it is proven that the combination of the time-reversal and inversion symmetries is important. These symmetries force $\theta\left(k_{\|}\right)$to obey

$$
\theta\left(k_{\|}\right)=-\theta\left(k_{\|}\right)-2 \pi \Delta^{I}\left(k_{\|}\right) \bmod 2 \pi,
$$

where

$$
\Delta^{I}\left(k_{\|}\right)=\sum_{n \in \text { filled }} \frac{1}{2 \pi \mathrm{i}} \int_{-\pi}^{\pi} \mathrm{d} k_{\perp}\left\langle u_{n k_{\|} k_{\perp}}\left|\hat{P}_{k}^{-1}\left(\partial_{k_{\perp}} \hat{P}_{k}\right)\right| u_{n k_{\|} k_{\perp}}\right\rangle
$$

with $\hat{P}_{\boldsymbol{k}}$ being the inversion symmetry operator satisfying $\hat{H}_{-k}=\hat{P}_{k} \hat{H}_{k} \hat{P}_{k}^{-1}$. Then, if $\Delta^{I}\left(k_{\|}\right)$is zero (because $\hat{P}_{k}$ has no $\boldsymbol{k}$ dependence, for instance) $\theta\left(k_{\|}\right)$becomes quantized to 0 or $\pi$, i.e., $Z_{2}$. Note that the inversion symmetry alone is sufficient for one-dimensional models, ${ }^{15}$ but it must be combined with the time-reversal symmetry for higher-dimensional cases. Note also that the reflection symmetry whose reflection plane is parallel to the edge alone can quantize $\theta\left(k_{\|}\right)$. In the case of fermionic SS model, the glide plane symmetry plays a role of the reflection plane symmetry. A derivation and discussions of Eq. (3) and related topics are given in Appendix.

A physical meaning of the $Z_{2}$ quantization can be understood from the fact that $\theta\left(k_{\|}\right)$has close relation to the electronic polarization. $^{32}$ The inversion or reflection symmetry gives restrictions for possible values of the electronic polarization, and these restrictions appear as the $Z_{2}$ quantization. However, a special attention is required in the case that the bulk 
symmetries are broken after introducing edges to the system. In our edge construction, edge shapes depend on the unit cell convention. Then, if we calculate $\theta\left(k_{\|}\right)$using a unit cell convention that leads to an edge breaking bulk inversion and glide plane symmetries, $\theta\left(k_{\|}\right)$is not necessarily quantized even if bulk system without edges has inversion and glide plane symmetries. This corresponds to the case that $\Delta^{I}\left(k_{\|}\right)$is noninteger. On the other hand, $\Delta^{I}\left(k_{\|}\right)=0$ basically happens when the system retains the global inversion symmetry even after the edges are introduced and the system becomes to have a ribbon shape, or in other words, when the two edges of the finite width system are equivalent.

\section{Bulk-edge correspondence}

The close relation between the appearance of edge states and $\theta\left(k_{\|}\right)$can be seen in Figs. 4(c) and 4(d). Namely, we find edge states for $k_{\|}$with $\theta\left(k_{\|}\right)=\pi \bmod 2 \pi$, while no edge states for $k_{\|}$with $\theta\left(k_{\|}\right)=0$. Since the $\pi$ jumps are related to the bulk Dirac points (as is explained later in Sec. III B), existence and nonexistence of the edge states is switched at the Dirac points projected to the edge. Here, we want to emphasize that, although $\theta\left(k_{\|}\right)$can be calculated only with bulk information, $\theta\left(k_{\|}\right)$apparently has an ability to capture the difference in edge terminations, i.e., difference between type 1 and type 2 edges. This is because $\theta\left(k_{\|}\right)$does depend on the choice of the basis set since its definition involves the Bloch wave functions, and different unit cell conventions are actually connected by a unitary transformation, i.e., a transformation of the basis set. The explicit transformation formula can be written as

$$
\theta^{\prime}\left(k_{\|}\right)=\theta\left(k_{\|}\right)-2 \pi \sum_{a} \epsilon_{a}^{\perp} \rho_{a}\left(k_{\|}\right),
$$

where

$$
\rho_{a}\left(k_{\|}\right) \equiv \sum_{n \in \text { filled }} \frac{1}{2 \pi} \int_{-\pi}^{\pi} \mathrm{d} k_{\perp}\left\langle u_{n k_{\|} k_{\perp}}\left|\mathcal{P}_{a}\right| u_{n k_{\|} k_{\perp}}\right\rangle .
$$

Here, $\epsilon_{a}^{\perp}$ denotes a component of a vector characterizing the unit cell transformation, $\mathcal{P}_{a}$ is an projection operator projecting on the site $a$ component, and $\rho_{a}\left(k_{\|}\right)$is $k_{\|}$resolved filling of site $a$. (See Appendix for details.) In our specific case, $\theta\left(k_{\|}\right)$ in type 1 and type 2 conventions are connected as

$$
\theta_{\text {type } 2}\left(k_{\|}\right)=\theta_{\text {type } 1}\left(k_{\|}\right)+2 \pi \rho_{1}\left(k_{\|}\right)
$$

with

$$
\rho_{1}\left(k_{x}\right)=\sum_{n \in \text { filled }} \frac{1}{2 \pi} \int_{-\pi}^{\pi} \mathrm{d} k_{y}\left\langle u_{n k_{x} k_{y}}\left|\mathcal{P}_{1}\right| u_{n k_{x} k_{y}}\right\rangle .
$$

As far as $t_{+}=t_{-}, \rho_{1}\left(k_{\|}\right)=0.5$ holds in our model by a symmetrical reason. Consequently, $\theta_{\text {type } 1}\left(k_{\|}\right)$and $\theta_{\text {type } 2}\left(k_{\|}\right)$ differ by $\pi$.

Intuitive understanding of this bulk-edge correspondence is possible with the help of adiabatic continuation when $\theta\left(k_{\|}\right)$ is quantized. We briefly explain this for the type 2 edge with parameters used in Fig. 4(d). Recall that the type 2 edge shows the edge states for $k_{\|}=\pi$ and no edge state for $k_{\|}=0$. If $k_{\|}$is fixed to $\pi, \boldsymbol{k}$ resolved Hamiltonian $\hat{H}_{\boldsymbol{k}}$ can be adiabatically deformed without gap closing and keeping $\theta\left(k_{\|}\right)$value to the Hamiltonian corresponding to $t_{x}=t_{y}=0$. Then, edge states are readily understood as dangling states appearing as

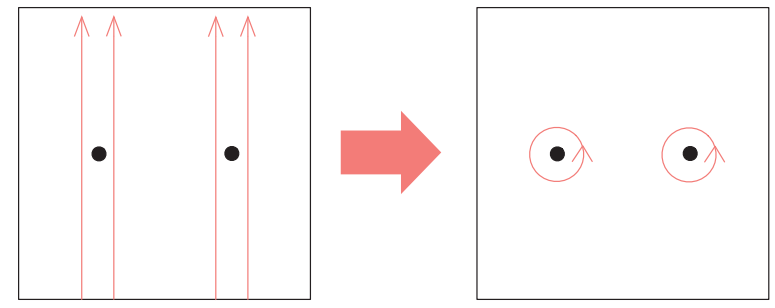

FIG. 5. (Color online) Schematic description of the relation between $\pi$ jumps in $\theta\left(k_{\|}\right)$and Dirac points.

a result of cutting remained diagonal bonds for type 2 edge. Importantly, the same adiabatic continuation cannot be applied to $k_{\|}=0$ case since it leads to the gap closing, which allows change in quantized $\theta\left(k_{\|}\right)$and leads to qualitative changes of the system properties. We have to use different adiabatic continuation, and that continuation should give Hamiltonian without dangling states for the type 2 edge.

\section{B. Further general applications of $Z_{2}$ Berry phase}

Possible applications of $Z_{2}$ Berry phases are not limited to the bulk-edge correspondence. First, the stability of massless Dirac fermions in two-dimensional systems can be clearly addressed using $Z_{2}$ Berry phase. In order to see this, we must realize that $\pi$ jump in $\theta\left(k_{\|}\right)$is directly related to a bulk Dirac fermion. If an infinitesimal change in $k_{\|}, k_{\|} \rightarrow k_{\|}+\delta k$ gives a finite change between $\theta\left(k_{\|}\right)$and $\theta\left(k_{\|}+\delta k\right)$, the electronic dispersion should have a singularity in the area enclosed by the two integration paths for $\theta\left(k_{\|}\right)$and $\theta\left(k_{\|}+\delta k\right)$, but, a massless Dirac fermion is nothing more than a singularity in the electronic dispersion. Furthermore, the value $\pi$ is exactly Berry phase acquired when the integration path encloses a Dirac point. The idea is described in Fig. 5 as a deformation of the integration path. Then, as far as the symmetries quantizing $\theta\left(k_{\|}\right)$are preserved, massless Dirac fermions are topologically stable, since $\pi$ jump cannot be suddenly removed by a small change in parameters when $\theta\left(k_{\|}\right)$is quantized to 0 or $\pi: \pi$ jump only disappears when two jumps are merged, or parameters themselves are discontinuously changed. Inversely speaking, if symmetries preserving $\theta\left(k_{\|}\right)$quantization is broken, massless Dirac fermion will be no longer stable. In fact, we have checked that when extra terms breaking the inversion and glide plane symmetries are added to the fermionic SS model, $\theta\left(k_{\|}\right)$ deviates from 0 or $\pi$, and a gap is induced at the Dirac point.

$Z_{2}$ Berry phase is also useful in making a criterion for the existence of massless Dirac fermions in a given model. ${ }^{34,35} \mathrm{As}$ discussed in Refs. 36 and 37, there is no need to explore the entire Brillouin zone to find out Dirac points, thanks to the $Z_{2}$ quantization. Instead, it is sufficient to check the values of $\theta\left(k_{\|}\right)$ at two $k_{\|} \mathrm{s}$, typically at $k_{\|}=0$ and $\pi$. If two $\theta\left(k_{\|}\right)$take different values, there must be at least one jump, or equivalently, Dirac point, as far as the quantization is retained.

\section{SUMMARY}

In summery, we have established a theory of the $Z_{2}$ Berry phase in general massless Dirac fermion systems. It is proved that ubiquitously existing symmetries like the time-reversal 
and the inversion symmetries, or the reflection symmetry are sufficient for quantizing the Berry phase defined with the Bloch wave functions. We have further shown the uses of $Z_{2}$ Berry phases, such as the bulk-edge correspondence and the topological stability of the Dirac cones. For the bulk-edge correspondence, we have shown that it is essential to connect the unit cell convention and the edge shapes appropriately in order to obtain the intimate relation between the Berry phase and the existence of the edge states. The ideas of $Z_{2}$ Berry phases have been applied to the fermionic Shastry-Sutherland model, which is revealed to be an interesting model by itself. Namely, the fermionic Shastry-Sutherland model has a rich phase diagrams, including phases with the quadratic band crossing point, the Dirac cones, and the pseudospin-1 Weyl fermion.

\section{ACKNOWLEDGMENTS}

This work is partly supported by Grants-in-Aid for Scientific Research Nos. 23340112, 25610101 (YH), and 23540460 (YH) from JSPS, and Science of Atomic Layers from MEXT, 25107005 (YH).

\section{APPENDIX: TRANSFORMATION FORMULAS AND QUANTIZATION OF THE BERRY PHASE}

\section{Hamiltonian and the Berry phase}

Our starting point is the Hamiltonian (1), which is the Hamiltonian for a tight-binding model of noninteracting spinless fermions:

$$
H=\sum_{a b} \sum_{\boldsymbol{r} \boldsymbol{r}^{\prime}} t_{a b}\left(\boldsymbol{r}-\boldsymbol{r}^{\prime}\right) c_{\boldsymbol{r} a}^{\dagger} c_{\boldsymbol{r}^{\prime} b}=\sum_{\boldsymbol{k}} \sum_{a b}\left(\hat{H}_{\boldsymbol{k}}\right)_{a b} c_{\boldsymbol{k} a}^{\dagger} c_{\boldsymbol{k} b} .
$$

Here,

$$
\left(\hat{H}_{\boldsymbol{k}}\right)_{a b}=\sum_{\boldsymbol{R}} t_{a b}(\boldsymbol{R}) \mathrm{e}^{\mathrm{i} \boldsymbol{k} \cdot \boldsymbol{R}},
$$

with $c_{\boldsymbol{r} a}=\frac{1}{\sqrt{N}} \sum_{k} \mathrm{e}^{-\mathrm{i} \boldsymbol{k} \cdot \boldsymbol{r}} c_{\boldsymbol{k} a}$, where $N$ is the total number of unit cells, $a$ and $b$ represent sublattices and orbitals, while $r$ and $\boldsymbol{r}^{\prime}$ represent lattice vectors, which are defined on a discrete lattice. Namely, using integers $n_{i}, \boldsymbol{r}$ can be written as $\boldsymbol{r}=$ $\sum_{i=1}^{d} n_{i} \boldsymbol{a}_{i}$, where $d$ is space dimension and $\boldsymbol{a}_{i}$ denote unit vectors of a specific model.

In the following, we consider the two-dimensional case and set $\boldsymbol{a}_{1}=(1,0)$ and $\boldsymbol{a}_{2}=(0,1)$ for simplicity, but further generalization is straightforward. We define the Berry phase $\theta\left(k_{\|}\right)$as

$$
\theta\left(k_{\|}\right)=-\mathrm{i} \sum_{n \in \text { filled }} \int_{-\pi}^{\pi} \mathrm{d} k_{\perp} A_{n}^{\perp}\left(k_{\|}, k_{\perp}\right),
$$

where

$$
A_{n}^{\perp}\left(k_{\|}, k_{\perp}\right)=\left\langle u_{n k_{\|} k_{\perp}}\left|\partial_{k_{\perp}}\right| u_{n k_{\|} k_{\perp}}\right\rangle .
$$

\section{Time-reversal symmetry}

The system is said to have the time-reversal symmetry if a relation

$$
\hat{H}_{-\boldsymbol{k}}=\mathcal{K} \hat{H}_{k} \mathcal{K}
$$

is satisfied. Here, $\mathcal{K}$ is a complex conjugate operator. In this case, if the eigenstate $\left|u_{n k}\right\rangle$ is nondegenerate, the eigenstates for $-\boldsymbol{k}$ and for $\boldsymbol{k}$ are connected as

$$
\left|u_{n-\boldsymbol{k}}\right\rangle=\mathcal{K}\left|u_{n \boldsymbol{k}}\right\rangle \mathrm{e}^{\mathrm{i} \phi_{n k}^{(t)}}
$$

Then, we have

$$
\begin{aligned}
A_{n}^{\perp}\left(-k_{\|},-k_{\perp}\right) & =\left\langle u_{n-k_{\|}-k_{\perp}}\left|\partial_{-k_{\perp}}\right| u_{n-k_{\|}-k_{\perp}}\right\rangle \\
& =-\left\langle\mathcal{K} u_{n k_{\|} k_{\perp}}\right| \mathrm{e}^{-\mathrm{i} \phi_{n k}^{(t)}} \partial_{k_{\perp}}\left(\mathrm{e}^{\mathrm{i} \phi_{n k}^{(t)}}\left|\mathcal{K} u_{n k_{\|} k_{\perp}}\right\rangle\right) \\
& =-\mathrm{i} \partial_{k_{\perp}} \phi_{n \boldsymbol{k}}^{(t)}-\left\langle\mathcal{K} u_{n k_{\|} k_{\perp}}\left|\partial_{k_{\perp}}\right| \mathcal{K} u_{n k_{\|} k_{\perp}}\right\rangle \\
& =-\mathrm{i} \partial_{k_{\perp}} \phi_{n \boldsymbol{k}}^{(t)}-\left\langle\partial_{k_{\perp}} u_{n k_{\|} k_{\perp}} \mid u_{n k_{\|} k_{\perp}}\right\rangle \\
& =-\mathrm{i} \partial_{k_{\perp}} \phi_{n \boldsymbol{k}}^{(t)}+\left\langle u_{n k_{\|} k_{\perp}}\left|\partial_{k_{\perp}}\right| u_{n k_{\|} k_{\perp}}\right\rangle \\
& =-\mathrm{i} \partial_{k_{\perp}} \phi_{n \boldsymbol{k}}^{(t)}+A_{n}^{\perp}\left(k_{\|}, k_{\perp}\right) .
\end{aligned}
$$

Here, we have used the relation $\partial_{-k_{\|}}=-\partial_{k_{\|}}$and the fact that $\mathcal{K}$ is an antiunitary operator. If we assume that the wave function is single valued in the Brillouin zone, the integration of $\partial_{k_{\perp}} \phi_{n k}^{(t)}$ with respect to $k_{\perp}$ from $-\pi$ to $\pi$ leads to integer multiples of $2 \pi$. Then, we obtain

$$
\begin{aligned}
\theta\left(-k_{\|}\right) & =-\mathrm{i} \sum_{n \in \text { filled }} \int_{-\pi}^{\pi} \mathrm{d} k_{\perp} A_{n}^{\perp}\left(-k_{\|}, k_{\perp}\right) \\
& =-\mathrm{i} \sum_{n \in \text { filled }} \int_{-\pi}^{\pi} \mathrm{d} k_{\perp} A_{n}^{\perp}\left(-k_{\|},-k_{\perp}\right) \\
& =\theta\left(k_{\|}\right)+2 \pi l .
\end{aligned}
$$

\section{Inversion symmetry}

The system is said to have the inversion symmetry if a relation

$$
\hat{H}_{-k}=\hat{P}_{k} \hat{H}_{k} \hat{P}_{k}^{-1}
$$

is satisfied with some unitary operator $\hat{P}_{\boldsymbol{k}}$. In this case, just as in the case of the time-reversal symmetry, the eigenstates for $\boldsymbol{-} \boldsymbol{k}$ and for $\boldsymbol{k}$ are connected as

$$
\left|u_{n-k}\right\rangle=\hat{P}_{k}\left|u_{n k}\right\rangle \mathrm{e}^{\mathrm{i} \phi_{n k}^{(I)}}
$$

if the eigenstate $\left|u_{n k}\right\rangle$ is nondegenerate. Then, we have

$$
\begin{aligned}
A_{n}^{\perp}\left(-k_{\|},-k_{\perp}\right)= & \left\langle u_{n-k_{\|}-k_{\perp}}\left|\partial_{-k_{\perp}}\right| u_{n-k_{\|}-k_{\perp}}\right\rangle \\
= & -\left\langle u_{n k_{\|} k_{\perp}}\right| \hat{P}_{\boldsymbol{k}}^{-1} \mathrm{e}^{-\mathrm{i} \phi_{n k}^{(I)}} \partial_{k_{\perp}}\left(\mathrm{e}^{\mathrm{i} \phi_{n k}^{(I)}} \hat{P}_{\boldsymbol{k}}\left|u_{n k_{\|} k_{\perp}}\right\rangle\right) \\
= & -\mathrm{i} \partial_{k_{\perp}} \phi_{n \boldsymbol{k}}^{(I)}-\left\langle u_{n k_{\|} k_{\perp}}\left|\hat{P}_{\boldsymbol{k}}^{-1}\left(\partial_{k_{\perp}} \hat{P}_{\boldsymbol{k}}\right)\right| u_{n k_{\|} k_{\perp}}\right\rangle \\
& -A_{n}^{\perp}\left(k_{\|}, k_{\perp}\right)
\end{aligned}
$$

and

$$
\begin{aligned}
\theta\left(-k_{\|}\right) & =-\mathrm{i} \sum_{n \in \text { filled }} \int_{-\pi}^{\pi} \mathrm{d} k_{\perp} A_{n}^{\perp}\left(-k_{\|}, k_{\perp}\right) \\
& =-\mathrm{i} \sum_{n \in \text { filled }} \int_{-\pi}^{\pi} \mathrm{d} k_{\perp} A_{n}^{\perp}\left(-k_{\|},-k_{\perp}\right) \\
& =-\theta\left(k_{\|}\right)+2 \pi l-2 \pi \Delta^{I}\left(k_{\|}\right),
\end{aligned}
$$




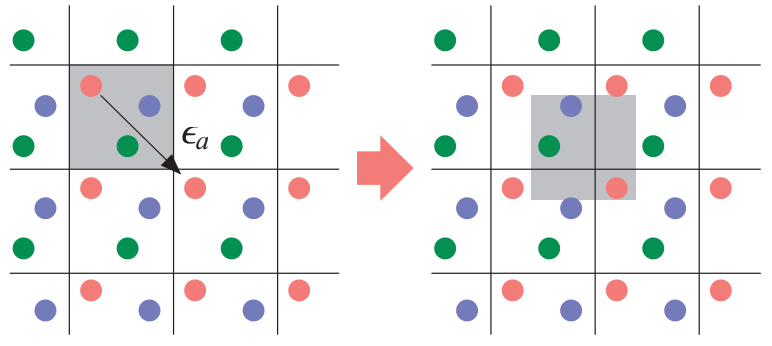

FIG. 6. (Color online) Schematic picture of the definition of $\boldsymbol{\epsilon}_{\boldsymbol{a}}$. Shaded regions denote the original (left) and updated (right) unit cells.

where

$$
\Delta^{I}\left(k_{\|}\right)=\sum_{n \in \text { filled }} \frac{1}{2 \pi \mathrm{i}} \int_{-\pi}^{\pi} \mathrm{d} k_{\perp}\left\langle u_{n k_{\|} k_{\perp}}\left|\hat{P}_{k}^{-1}\left(\partial_{k_{\perp}} \hat{P}_{k}\right)\right| u_{n k_{\|} k_{\perp}}\right\rangle .
$$

If Eq. (A12) is combined with Eq. (A8), we have

$$
\theta\left(k_{\|}\right)=-\theta\left(k_{\|}\right)+2 \pi l-2 \pi \Delta^{I}\left(k_{\|}\right),
$$

which leads to the $Z_{2}$ quantization of $\theta\left(k_{\|}\right)$when $\Delta^{I}\left(k_{\|}\right)$is zero or any integer.

\section{Reflection plane parallel to the edge}

A relation similar to Eq. (A14) can be derived also in the case that the system has a reflection plane parallel to the direction of $k_{\|}$, i.e., in the case that there exists $\hat{R}_{k}^{\|}$fulfilling

$$
\hat{H}_{k_{\|},-k_{\perp}}=\hat{R}_{\boldsymbol{k}}^{\|} \hat{H}_{k_{\|}, k_{\perp}} \hat{R}_{\boldsymbol{k}}^{\|-1} .
$$

In the similar way as in the previous section, we can derive

$$
\theta\left(k_{\|}\right)=-\theta\left(k_{\|}\right)+2 \pi l-2 \pi \Delta^{\|}\left(k_{\|}\right),
$$

where

$$
\Delta^{\|}\left(k_{\|}\right)=\sum_{n \in \text { filled }} \frac{1}{2 \pi \mathrm{i}} \int_{-\pi}^{\pi} \mathrm{d} k_{\perp}\left\langle u_{n k_{\|} k_{\perp}}\left|\hat{R}_{k}^{\|-1}\left(\partial_{k_{\perp}} \hat{R}_{k}^{\|}\right)\right| u_{n k_{\|} k_{\perp}}\right\rangle .
$$

Equation (A16) causes $Z_{2}$ quantization of $\theta\left(k_{\|}\right)$if $\Delta^{\|}\left(k_{\|}\right)$is an integer.

\section{Unit cell convention}

If the model contains multiple sublattices in a unit cell, the way to construct a unit cell is not unique. Namely, there are several possible unit cell conventions. Modification of the unit cell can be achieved by reinterpreting some sublattices in a unit cell, say at $\boldsymbol{r}$, as sublattices belonging to a different unit cell, say at $\boldsymbol{r}+\boldsymbol{\epsilon}_{a}$. (A schematic description of $\boldsymbol{\epsilon}_{a}$ is given in Fig. 6.) This modification can be captured by replacing $t_{a b}\left(\boldsymbol{r}-\boldsymbol{r}^{\prime}\right)$ as

$$
t_{a b}\left(\boldsymbol{r}-\boldsymbol{r}^{\prime}\right) \longrightarrow t_{a b}\left(\boldsymbol{r}+\boldsymbol{\epsilon}_{a}-\boldsymbol{r}^{\prime}-\boldsymbol{\epsilon}_{b}\right) .
$$

Then, $\hat{H}_{k}$ is transformed as

$$
\begin{aligned}
\left(\hat{H}_{\boldsymbol{k}}\right)_{a b} \longrightarrow\left(\hat{H}_{\boldsymbol{k}}^{\prime}\right)_{a b} & =\sum_{\boldsymbol{R}} t_{a b}\left(\boldsymbol{R}+\boldsymbol{\epsilon}_{a}-\boldsymbol{\epsilon}_{b}\right) \mathrm{e}^{\mathrm{i} \boldsymbol{k} \cdot \boldsymbol{R}} \\
& =\sum_{\boldsymbol{R}} \mathrm{e}^{-\mathrm{i} \boldsymbol{k} \cdot \boldsymbol{\epsilon}_{a}} t_{a b}(\boldsymbol{R}) \mathrm{e}^{\mathrm{i} \boldsymbol{k} \cdot \boldsymbol{R}} \mathrm{e}^{\mathrm{i} \boldsymbol{k} \cdot \boldsymbol{\epsilon}_{b}},
\end{aligned}
$$

which can be written in a compact form as

$$
\hat{H}_{k} \longrightarrow \hat{H}_{k}^{\prime}=\hat{U}_{k} \hat{H}_{k} \hat{U}_{k}^{\dagger},
$$

where

$$
\left(\hat{U}_{\boldsymbol{k}}\right)_{a b}=\delta_{a b} \mathrm{e}^{-\mathrm{i} \boldsymbol{k} \cdot \boldsymbol{\epsilon}_{a}} .
$$

Then, the eigenstates of $\hat{H}_{k}$ and $\hat{H}_{k}^{\prime}$ are connected as

$$
\left|u_{n k}^{\prime}\right\rangle=\hat{U}_{k}\left|u_{n k}\right\rangle .
$$

Using Eq. (A22), we have

$$
\begin{aligned}
A_{n}^{\perp \prime}\left(k_{\|}, k_{\perp}\right) & =\left\langle u_{n k_{\|} k_{\perp}}^{\prime}\left|\partial_{k_{\perp}}\right| u_{n k_{\|} k_{\perp}}^{\prime}\right\rangle \\
& =\left\langle u_{n k_{\|} k_{\perp}}\right| \hat{U}_{k_{\|} k_{\perp}}^{\dagger} \partial_{k_{\perp}}\left(\hat{U}_{k_{\|} k_{\perp}}\left|u_{n k_{\|} k_{\perp}}\right\rangle\right) \\
& =-\mathrm{i} \sum_{a} \epsilon_{a}^{\perp}\left\langle u_{n k_{\|} k_{\perp}}\left|\mathcal{P}_{a}\right| u_{n k_{\|} k_{\perp}}\right\rangle+A_{n}^{\perp}\left(k_{\|}, k_{\perp}\right),
\end{aligned}
$$

where $\mathcal{P}_{a}$ is a projection operator projecting on a component $a$, and $\epsilon_{a}^{\perp}$ denotes the component of $\boldsymbol{\epsilon}_{a}$ perpendicular to the direciton of $k_{\|}$. We have used a relation

$$
\hat{U}_{k_{\|} k_{\perp}}^{\dagger}\left(\partial_{k_{\perp}} \hat{U}_{k_{\|} k_{\perp}}\right)=-\mathrm{i} \sum_{a} \epsilon_{a}^{\perp} \mathcal{P}_{a},
$$

which can be derived from the definition, namely, Eq. (A21). Then, we finally obtain a transformation formula

$$
\theta^{\prime}\left(k_{\|}\right)=\theta\left(k_{\|}\right)-2 \pi \sum_{a} \epsilon_{a}^{\perp} \rho_{a}\left(k_{\|}\right),
$$

where

$$
\rho_{a}\left(k_{\|}\right) \equiv \sum_{n \in \text { filled }} \frac{1}{2 \pi} \int_{-\pi}^{\pi} \mathrm{d} k_{\perp}\left\langle u_{n k_{\Downarrow} k_{\perp}}\left|\mathcal{P}_{a}\right| u_{n k_{\Downarrow} k_{\perp}}\right\rangle .
$$

For the specific case of the fermionic Shastry-Sutherland model in the main body, the transformation from the type 1 to type 2 unit cell convention corresponds to the case of

$$
\boldsymbol{\epsilon}_{a}=\left\{\begin{array}{ll}
\hat{\boldsymbol{x}}-\hat{\boldsymbol{y}} & (a=1) \\
0 & (a=2,3,4)
\end{array} .\right.
$$

Then, from Eqs. (A25) and (A27), we have

$$
\theta_{\text {type } 2}\left(k_{\|}\right)=\theta_{\text {type } 1}\left(k_{\|}\right)+2 \pi \rho_{1}\left(k_{\|}\right),
$$

when the edge is parallel to the $x$ axis, i.e., $k_{\|}=k_{x}$. When $t_{+}=$ $t_{-}, \rho_{1}\left(k_{\|}\right)$is determined by the symmetry. Namely, the timereversal symmetry, the inversion symmetry, the glide plane symmetry whose glide plane is parallel to the $x$ and $y$ axes leads to the relations $\rho_{1}\left(k_{\|}\right)=\rho_{1}\left(-k_{\|}\right), \rho_{1}\left(k_{\|}\right)=\rho_{2}\left(-k_{\|}\right), \rho_{1}\left(k_{\|}\right)=$ $\rho_{3}\left(-k_{\|}\right)$, and $\rho_{1}\left(k_{\|}\right)=\rho_{4}\left(k_{\|}\right)$, respectively. These relations are summarized to $\rho_{1}\left(k_{\|}\right)=\rho_{2}\left(k_{\|}\right)=\rho_{3}\left(k_{\|}\right)=\rho_{4}\left(k_{\|}\right)$, which gives $\rho_{1}\left(k_{\|}\right)=0.5$ for the half-filled case. Then, we have

$$
\theta_{\text {type } 2}\left(k_{\|}\right)=\theta_{\text {type } 1}\left(k_{\|}\right)+\pi .
$$


*kariyado.t.gf@u.tsukuba.ac.jp

${ }^{1}$ K. S. Novoselov, A. K. Geim, S. V. Morozov, D. Jiang, M. I. Katsnelson, I. V. Grigorieva, S. V. Dubonos, and A. A. Firsov, Nature (London) 438, 197 (2005).

${ }^{2}$ S. Katayama, A. Kobayashi, and Y. Suzumura, J. Phys. Soc. Jpn. 75, 054705 (2006).

${ }^{3}$ M. Z. Hasan and C. L. Kane, Rev. Mod. Phys. 82, 3045 (2010).

${ }^{4}$ X.-L. Qi and S.-C. Zhang, Rev. Mod. Phys. 83, 1057 (2011).

${ }^{5}$ J. Moore, Nat. Phys. 5, 378 (2009).

${ }^{6}$ N. Goldman, A. Kubasiak, A. Bermudez, P. Gaspard, M. Lewenstein, and M. A. Martin-Delgado, Phys. Rev. Lett. 103, 035301 (2009).

${ }^{7}$ L. Tarruell, D. Greif, T. Uehlinger, G. Jotzu, and T. Esslinger, Nature (London) 483, 302 (2012).

${ }^{8}$ M. Fujita, K. Wakabayashi, K. Nakada, and K. Kusakabe, J. Phys. Soc. Jpn. 65, 1920 (1996).

${ }^{9}$ S. Ryu and Y. Hatsugai, Phys. Rev. Lett. 89, 077002 (2002).

${ }^{10}$ T. Kariyado and M. Ogata, J. Phys. Soc. Jpn. 81, 064701 (2012).

${ }^{11}$ Y. Hatsugai, Phys. Rev. Lett. 71, 3697 (1993).

${ }^{12}$ Y. Hatsugai, Solid State Commun. 149, 1061 (2009).

${ }^{13}$ P. Delplace, D. Ullmo, and G. Montambaux, Phys. Rev. B 84, 195452 (2011).

${ }^{14}$ D. J. Thouless, M. Kohmoto, M. P. Nightingale, and M. den Nijs, Phys. Rev. Lett. 49, 405 (1982).

${ }^{15}$ J. Zak, Phys. Rev. Lett. 62, 2747 (1989).

${ }^{16}$ Y. Hatsugai, J. Phys. Soc. Jpn. 75, 123601 (2006).

${ }^{17}$ Y. Hatsugai, New J. Phys. 12, 065004 (2010).

${ }^{18}$ T. Fukui, K.-I. Imura, and Y. Hatsugai, J. Phys. Soc. Jpn. 82, 073708 (2013).

${ }^{19}$ M. V. Berry, Proc. R. Soc. A 392, 45 (1984).
${ }^{20}$ B. S. Shastry and B. Sutherland, Physica B+C 108, 1069 (1981).

${ }^{21}$ B. S. Shastry and B. Kumer, Prog. Theor. Phys. Suppl. 145, 1 (2002).

${ }^{22}$ S. Miyahara and K. Ueda, J. Phys.: Condens. Matter 15, R327 (2003).

${ }^{23}$ I. Maruyama, S. Tanaya, M. Arikawa, and Y. Hatsugai, J. Phys.: Conf. Ser. 320, 012019 (2011).

${ }^{24}$ H. Kageyama, K. Yoshimura, R. Stern, N. V. Mushnikov, K. Onizuka, M. Kato, K. Kosuge, C. P. Slichter, T. Goto, and Y. Ueda, Phys. Rev. Lett. 82, 3168 (1999).

${ }^{25}$ K. Sun, H. Yao, E. Fradkin, and S. A. Kivelson, Phys. Rev. Lett. 103, 046811 (2009).

${ }^{26}$ E. Dagotto, E. Fradkin, and A. Moreo, Phys. Lett. B 172, 383 (1986).

${ }^{27}$ Z. Lan, N. Goldman, and P. Öhberg, Phys. Rev. B 85, 155451 (2012).

${ }^{28}$ Y. Yamashita, M. Tomura, Y. Yanagi, and K. Ueda, Phys. Rev. B 88, 195104 (2013).

${ }^{29}$ J. Liu, N. Trivedi, Y. Lee, B. N. Harmon, and J. Schmalian, Phys. Rev. Lett. 99, 227003 (2007).

${ }^{30}$ K. Sun, W. V. Liu, A. Hemmerich, and S. Das Sarma, Nat. Phys. 8, 67 (2012).

${ }^{31}$ G. Montambaux, F. Piéchon, J.-N. Fuchs, and M. O. Goerbig, Eur. Phys. J. B 72, 509 (2009).

${ }^{32}$ R. D. King-Smith and D. Vanderbilt, Phys. Rev. B 47, 1651 (1993).

${ }^{33}$ T. Hirano, H. Katsura, and Y. Hatsugai, Phys. Rev. B 78, 054431 (2008).

${ }^{34}$ C. Herring, Phys. Rev. 52, 365 (1937).

${ }^{35}$ K. Asano and C. Hotta, Phys. Rev. B 83, 245125 (2011).

${ }^{36}$ T. Mori, J. Phys. Soc. Jpn. 82, 034712 (2013).

${ }^{37}$ F. Piéchon and Y. Suzumura, J. Phys. Soc. Jpn. 82, 033703 (2013). 